\title{
La cadena productiva de la industria de la madera de Santiago del Estero (Argentina)
}

The productive chain of the wood industry of Santiago del Estero (Argentina)

\section{A cadeia produtiva da indústria da madeira de} Santiago del Estero (Argentina)

\author{
Rolando Schimpf ${ }^{1}$ \\ Rocío Sánchez Ugalde ${ }^{2}$ \\ Myriam Ludueña ${ }^{3}$ \\ Marta Coronel de Renolfi ${ }^{4}$
}

Recibido: junio 5 del 2018

Aprobado: octubre 15 del 2018

Disponible en línea: enero 15 del 2019

Cómo citar este artículo:

R. Schimpf, R. Sánchez Ugalde, M. Ludueña y M. Coronel de Renolfi, "La cadena productiva de la industria de la madera de Santiago del Estero (Argentina)", Revista

Ingeniería Solidaria, vol. 25, n. ${ }^{\circ} 1,2019$.

DOI: https://doi.org/10.16925/2357-6014.2019.01.04

Artículo de investigación. https://doi.org/10.16925/2357-6014.2019.01.04

1 Universidad Nacional de Santiago del Estero, Santiago del Estero, Argentina ORCID: https://orcid.org/0000-0002-8116-1230

2 Universidad Nacional de Santiago del Estero, Santiago del Estero, Argentina ORCID: https://orcid.org/0000-0001-5816-0304

3 Universidad Nacional de Santiago del Estero, Santiago del Estero, Argentina ORCID: https://orcid.org/0000-0002-0528-6867

4 Universidad Nacional de Santiago del Estero, Santiago del Estero, Argentina ORCID: https://orcid.org/0000-0001-9176-1689

Correo electrónico: mrenolfi@gmail.com 


\title{
Resumen
}

Introducción: este artículo es producto de la "Investigación de mercado de la madera de especies para uso sólido. El caso de las especies forestales maderables de Santiago del Estero", desarrollada en la Universidad Nacional de Santiago del Estero en el periodo 2016-2017. El objetivo del trabajo fue describir la cadena productiva de la industria forestal de la provincia de Santiago del Estero, con énfasis en la cadena de la madera y el mueble, e identificar los factores críticos para su desempeño.

Metodología: para examinar la cadena productiva se aplicó el análisis diagnóstico mediante revisión bibliográfica y recolección de información en terreno; y a fin de identificar factores críticos de la cadena, se efectuó un análisis FODA.

Resultados: en la cadena foresto-industrial existe una estrecha conexión entre el eslabón de la producción primaria y los siguientes, dado que los eslabones de la primera y segunda transformaciones utilizan la madera de origen local como fuente principal de aprovisionamiento.

Conclusiones: la cadena foresto-industrial con origen en los bosques nativos tiene continuidad en todos los eslabones productivos. El alto grado de informalidad con el que se desarrollan las actividades productivas, así como la fuerte cultura individualista en las conductas de los actores directos, no permiten generar procesos de integración. Se identifican debilidades y amenazas en todo el eslabonamiento de la cadena. No obstante, existen fortalezas y oportunidades para potenciar y capitalizar.

Originalidad: no existen antecedentes de trabajos publicados sobre el tema para la provincia.

Limitaciones: insuficiente información pública y privada sobre el tema.

Palabras clave: cadena productiva, industria maderera, fortalezas y debilidades, pymes madereras.

\begin{abstract}
Introduction: this article is a product of research. The case of the forest species of Santiago del Estero, developed at the National University of Santiago del Estero in the period 2016-2017. The objective of the work was to describe the productive chain of the forest industry of the province of Santiago del Estero, with emphasis on the chain of wood and furniture, and identify the critical factors for its performance.

Methodology: to examine the productive chain, it is applied to the diagnostic analysis through bibliographic review and information gathering in the field; and in order to identify critical factors of the chain, a SWOT analysis was carried out.
\end{abstract}

Results: in the forestry-industrial chain there is a close connection between the link of primary production and the following ones, given that the links of the first and second transformations of wood of local origin as the main source of supply.

Conclusions: the forest-industrial chain originating in native forests has continuity in all the productive links. The high degree of information that refers to the productive activities, as well as the individualistic culture, the behaviors of the direct actors, do not allow generating integration processes. Weaknesses and threats are identified throughout the chain's linkage. However, there are strengths and opportunities to enhance and capitalize.

Originality: there is no background of published works on the subject for the province.

Limitations: insufficient public and private information on the subject.

Keywords: productive chain, wood industry, strengths and weaknesses, timber SMEs.

\section{Resumo}

Introdução: este artigo é produto da pesquisa "Pesquisa de mercado da madeira de espécies para uso sólido. o caso das espécies florestais madeiráveis de Santiago del Estero", desenvolvida na Universidad Nacional de 
Santiago del Estero, no período de 2016-2017. O objetivo do trabalho foi descobrir a cadeia produtiva da indústria florestal da província de Santiago del Estero, com ênfase na cadeia da madeira e do móvel, e identificar os fatores críticos para seu desempenho.

Metodologia: para examinar a cadeia produtiva, aplicou-se a análise diagnóstica por meio de revisão bibliográfica e levantamento de informação em terreno. Também efetuou-se uma análise swot (ou FOFA) a fim de identificar fatores críticos da cadeia.

Resultados: na cadeia floresto-industrial existe uma estreita conexão entre o elo da produção primária e os seguintes, visto que os elos da primeira e da segunda transformação utilizam madeira de origem local como fonte principal de abastecimento.

Conclusões: a cadeia floresto-industrial com origem nos bosques nativos tem continuidade em todos os elos produtivos. 0 alto grau de informalidade com que as atividades produtivas são desenvolvidas, bem como a forte cultura individualista nas condutas dos atores diretos, não permitem gerar processos de integração. Identificam-se fraquezas e ameaças em todo o encadeamento. No entanto, existem forças e oportunidades para potencializar e capitalizar.

Originalidade: não existem antecedentes de trabalhos publicados sobre o tema para a província.

Limitações: insuficiente informação pública e privada sobre o tema.

Palavras-chave: cadeia produtiva, indústria madeireira, forças e fraquezas, PMEs madeireiras.

\section{Introducción}

Según el Ordenamiento Territorial de Bosques Nativos (OTBN), Argentina cuenta con 53,6 millones de hectáreas de bosque nativo [1] y 1,3 millones de bosques cultivados [2].

Dentro del sector forestal argentino, la industria de la madera es el primer destino de la producción del bosque nativo e implantado, y el principal segmento generador del producto interno bruto (PIB) sectorial, con una participación del 49 \%. Esta industria consiste en la transformación física o mecánica de la madera, y está conformada por los siguientes subsectores: aserrados, tableros de fibra y de partículas, compensados y alistonados, envases, palets, postes y rodrigones, pisos, revestimientos, aberturas, carpinterías y muebles, y otras manufacturas.

El sector de la madera y muebles está compuesto por 8.441 unidades productivas, de las cuales el 98,9\% son pymes. Su participación en el valor agregado industrial fue del 7,4 \% en 2016. El sector emplea en forma directa y formal a 90.747 personas, lo que explica el 7,3 \% del empleo industrial argentino [3].

Las industrias de primera y segunda transformación de la madera se asientan de forma preponderante en las cercanías de los recursos forestales, ubicados principalmente en las zonas de la Mesopotamia y Buenos Aires, debido a economías de localización asociadas a los costos de transporte. Los eslabones más avanzados en la cadena de valor, en especial los de consumo final como muebles, se instalan cerca 
La cadena productiva de la industria de la madera de Santiago del Estero (Argentina)

de los centros de consumo (Buenos Aires, Santa Fe y Córdoba), que cuentan con mayores ingresos per cápita y mayor disponibilidad de mano de obra calificada. En las otras regiones del país existe una importante cantidad de aserraderos, especialmente de maderas nativas, y pequeñas producciones de muebles y otros productos madereros.

En particular, Santiago del Estero cuenta con 7,1 millones de ha de bosques nativos [1] que representan el 13,2 \% del total del país, y es la más importante fuente de abastecimiento de materia prima a la industria forestal local. La provincia posee, además, unas 5.580 ha de bosques implantados, en edad inmadura [2].

El informe del Censo Nacional de Aserraderos (CNA) [4] señala que la industria forestal de la provincia comprende dos subsectores bien diferenciados: la industria primaria, que procesa rollizos y elabora durmientes y madera aserrada para el campo; y la secundaria, que abarca la elaboración de aberturas, muebles, pisos de madera, implementos para la ganadería y otros productos de carpintería. Dicho censo identifica tres zonas madereras: norte (comprende los departamentos de Pellegrini, Copo y Alberdi); centro (departamentos de Capital, Banda, Río Hondo, Figueroa, Moreno, Ibarra, Sarmiento, San Martín, Robles, Silípica y Guasayán) y zona sur (departamentos de Choya, Loreto, Atamisqui, Avellaneda, Taboada, Ojo de Agua, Salavina, Quebrachos, Aguirre, Mitre, Belgrano y Rivadavia).

Sánchez Ugalde [5], por su parte, señala que Santiago del Estero cuenta con tres polos transformadores, bien diferenciados entre sí. Existe un nodo industrial en la zona centro, en los departamentos Capital y Banda (nodo Capital-Banda); otro en la zona sur, en el departamento Loreto (nodo Loreto); y en el tercero, situado en la zona norte de la provincia (nodo Noreste), se encuentra la mayor parte de los recursos forestales nativos y aglutina aserraderos en los departamentos Copo y Alberdi.

El Plan Estratégico 2020 de Santiago del Estero [6] consigna que el sector de la industria de la madera cuenta con 280 unidades productivas de diversos tamaños. Si bien las empresas se encuentran concentradas principalmente en los departamentos de Capital, Banda, Loreto, Copo, Alberdi y Choya, la actividad se encuentra atomizada y cuenta con unidades productivas en casi toda la provincia. Las carpinterías que fabrican muebles y aberturas se localizan, generalmente, en las cabeceras de departamento, y los aserraderos en el interior de las mismas. La materia prima forestal surge, básicamente, del aprovechamiento del bosque nativo y es industrializada (salvo excepciones puntuales) mediante sistemas productivos de bajo nivel tecnológico y escasa diversificación de la producción. Las alternativas de solución a estas limitaciones son los programas que promuevan la incorporación de tecnologías asociadas a 
escalas competitivas y a la diversificación productiva, y traten de vincular esta última al ciclo de actividad del sector de la construcción.

El mercado local de productos madereros está conformado por una gran variedad de productos de diversos grados de valor agregado, que provienen de los mencionados polos madereros de Santiago del Estero y otras provincias. Los precios de los productos que provienen de otras provincias son el resultado de sus contextos económicos y de políticas de fortalecimiento y desarrollo, conforme a las variables y factores propios del sector en cada provincia, favoreciendo la competitividad de dichos productos. En este sentido, para el mercado santiagueño resulta necesario el fortalecimiento de las pymes madereras en el camino hacia la competitividad.

Cuevas [7] señala que el análisis de cadenas productivas sirve como instrumento que permite identificar los puntos críticos que frenan la competitividad de un sector, para luego definir e impulsar estrategias concertadas entre los principales actores involucrados. Tanto para su análisis como para la formulación de estrategias de intervención, es necesario identificar los diferentes eslabones que la componen.

\subsection{Cadena productiva: marco teórico}

La cadena productiva es un sistema constituido por actores interrelacionados y por una sucesión de operaciones de producción, transformación y comercialización de un producto o grupo de productos en un entorno determinado. La cadena productiva se define como el conjunto de agentes y actividades económicas que intervienen en un proceso productivo desde la provisión de insumos y materias primas, su transformación y producción de bienes intermedios y finales, hasta la comercialización en los mercados internos y externos, incluyendo proveedores de servicios, sector público, instituciones de asistencia técnica y organismos de financiamiento [8].

En algunas ocasiones, el término 'cadena productiva' se sustituye por otro concepto utilizado en el mundo de los negocios para mejorar la competitividad: 'cadena de valor'. Sin embargo, el primero mantiene importantes diferencias con el segundo: mientras que en la cadena productiva los actores buscan el beneficio económico individual y compiten por ello, en la cadena de valor el objetivo es el mutuo apoyo entre los actores a través de cooperación, comunicación y coordinación [8].

Según Castro [9], la investigación de las cadenas productivas comprende dos etapas: el análisis diagnóstico y el análisis pronóstico. En la primera fase se investiga el desempeño pasado y actual de la cadena productiva; y en la segunda, el comportamiento futuro de esta. 
El análisis diagnóstico comprende una secuencia de pasos: inicia en el modelaje de la cadena, con la descripción detallada de cada segmento; continúa con la reseña del ambiente organizacional, que consiste en una matriz de organizaciones públicas, privadas y no gubernamentales; y finaliza con la definición del contexto institucional, en el cual interactúa un conjunto de políticas, planes, programas y leyes en el que se desarrolla la actividad. Este análisis tiene por finalidad caracterizar e identificar factores críticos, los cuales pueden ser cualquier variable que afecte de forma positiva o negativa el desempeño y expansión de la cadena: restricciones, limitaciones, retos y oportunidades.

El análisis pronóstico se efectúa con la información generada por el análisis diagnóstico, con el fin de proyectar el comportamiento futuro de los factores críticos.

\subsection{Objetivo del trabajo}

El objetivo general del trabajo presentado aquí fue describir y caracterizar la cadena productiva de la industria forestal de la provincia de Santiago del Estero, con énfasis en la cadena de la madera y el mueble, e identificar los factores críticos para el desempeño de esta.

\subsection{Antecedentes de investigación}

A nivel local, son insuficientes los antecedentes sobre el análisis de las cadenas productivas de Santiago del Estero. El Plan Estratégico 2020 [6] detalla las cadenas productivas líderes del desarrollo industrial de Santiago del Estero -textil, bioenergía, frutihortícola, cárnica e insumos para la construcción- y dedica escasas páginas a describir las cadenas secundarias, entre ellas la foresto-industrial. El reciente Censo Nacional de Aserraderos [4], si bien cubre un importante vacío de información, solo reporta datos acerca del conjunto del sector a nivel provincial. El trabajo más actual es el de Sánchez Ugalde [5], que efectuó un análisis diagnóstico en el año 2012.

A nivel nacional, las instituciones oficiales vinculadas al sector forestal publican, en general, información agregada. Entre las referencias más actuales se pueden citar el análisis de diagnóstico sectorial Madera y Muebles [10], el análisis prospectivo del complejo foresto-industrial [11], y el informe de cadenas de valor del sector forestal, papel y muebles [12].

Existen antecedentes del tema de autores latinoamericanos, entre los que se pueden citar el trabajo de Rodríguez Zúñiga et al. [13] en México, en el que se realiza un análisis estratégico de la cadena productiva forestal en la región de la Reserva 
de la Biosfera de la Mariposa Monarca; el de Padilla Puerta [14], que propone una formulación de la cadena productiva forestal en una región de Colombia; el de Salas Navarro [15], que analiza la cadena de abastecimiento del sector madera y muebles de la ciudad de Barranquilla; y el de Lima [16], que caracteriza la cadena de producción y comercialización de la industria forestal en Chile.

\section{Materiales y métodos}

Para examinar la cadena productiva de la industria de la madera se aplicó el análisis diagnóstico, descrito por Cuevas [7] y Castro [9]. El mismo consistió en la descripción de sus eslabones y los actores presentes en cada uno de ellos, mediante revisión bibliográfica y recolección de información en terreno. Después se efectuó un análisis FODA, según la metodología propuesta por David [17] y Ponce [18], que permitió identificar restricciones y cuellos de botella, así como desafíos y oportunidades para el crecimiento de la cadena.

La recolección de información consistió, básicamente, en la búsqueda de fuentes secundarias (publicaciones y bases de datos). También se recurrió a la obtención de información primaria mediante la aplicación de encuestas, esto es, entrevistas con aplicación de un cuestionario. El universo de análisis fue definido como el sector de la industria de transformación física de la madera (industria de la madera y el mueble), conformado por establecimientos emplazados en los tres nodos madereros citados por Sánchez Ugalde [5]: Capital y Banda, Loreto y Noreste (Monte Quemado y Campo Gallo). La figura 1 muestra los sitios de estudio.

Las entrevistas se realizaron in situ durante el segundo semestre de 2016 y el primero de 2017, a 90 titulares de pymes madereras. Se aplicó un muestreo por conveniencia que consiste en la elección, por métodos no aleatorios, de una muestra cuyas características sean similares a las de la población objetivo. 


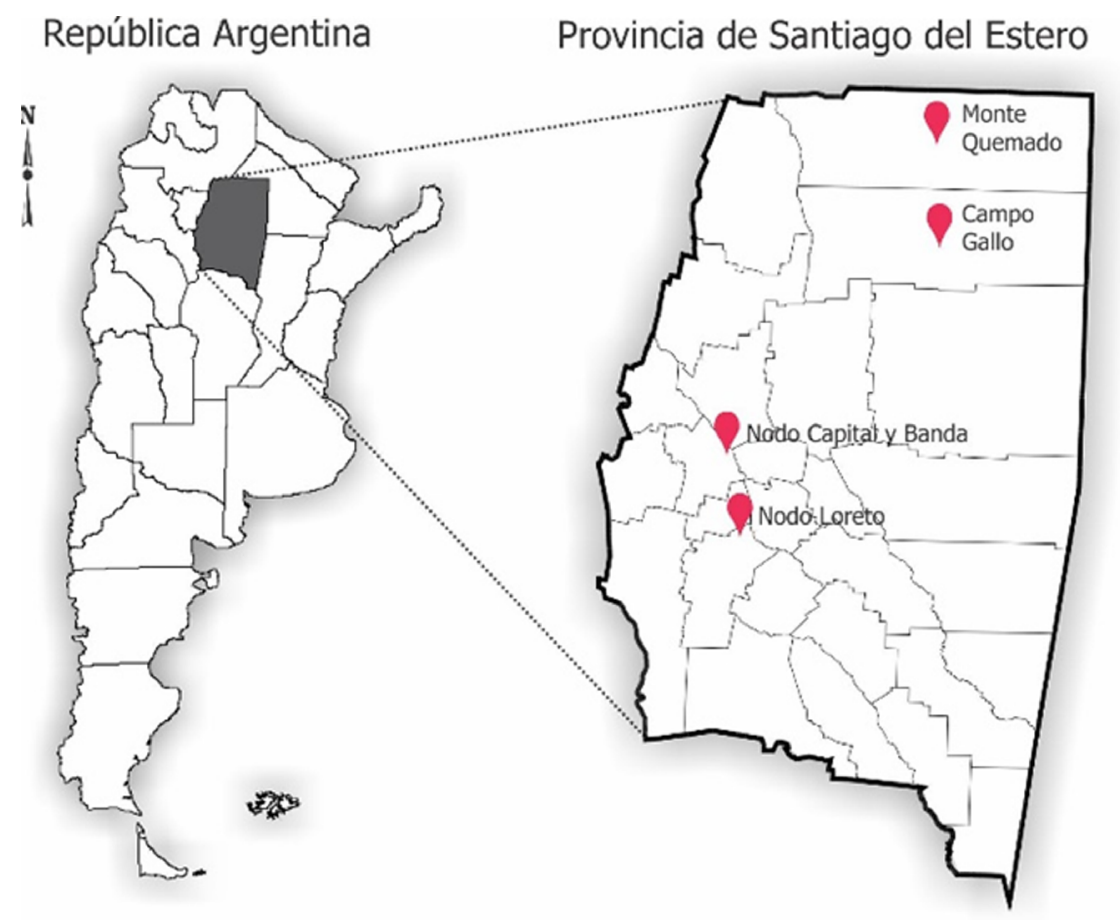

Figura 1. Santiago del Estero. Ubicación de los sitios de estudio.

Fuente: elaboración propia

\section{Resultados y discusión}

\subsection{Los eslabones de la cadena forestal en Santiago del Estero}

La cadena productiva en el sector foresto-industrial de Argentina tiene la estructura que se muestra en la figura 2.

En la figura se puede apreciar que hay dos tipos de fuentes proveedoras de materias primas: los bosques implantados y los nativos. Los principales eslabones productivos en la cadena forestal se corresponden con la producción primaria, la primera y la segunda transformación, cuyos productos son destinados a la construcción, a la producción de energía o a la fabricación de bienes intermedios o finales, para ser consumidos en el mercado interno o externo.

En cuanto a la distribución territorial de la cadena, Idígoras [11] informa acerca de la activa participación de algunas regiones del país como la mesopotámica, donde se encuentran las cadenas foresto-industriales más desarrolladas del país. 


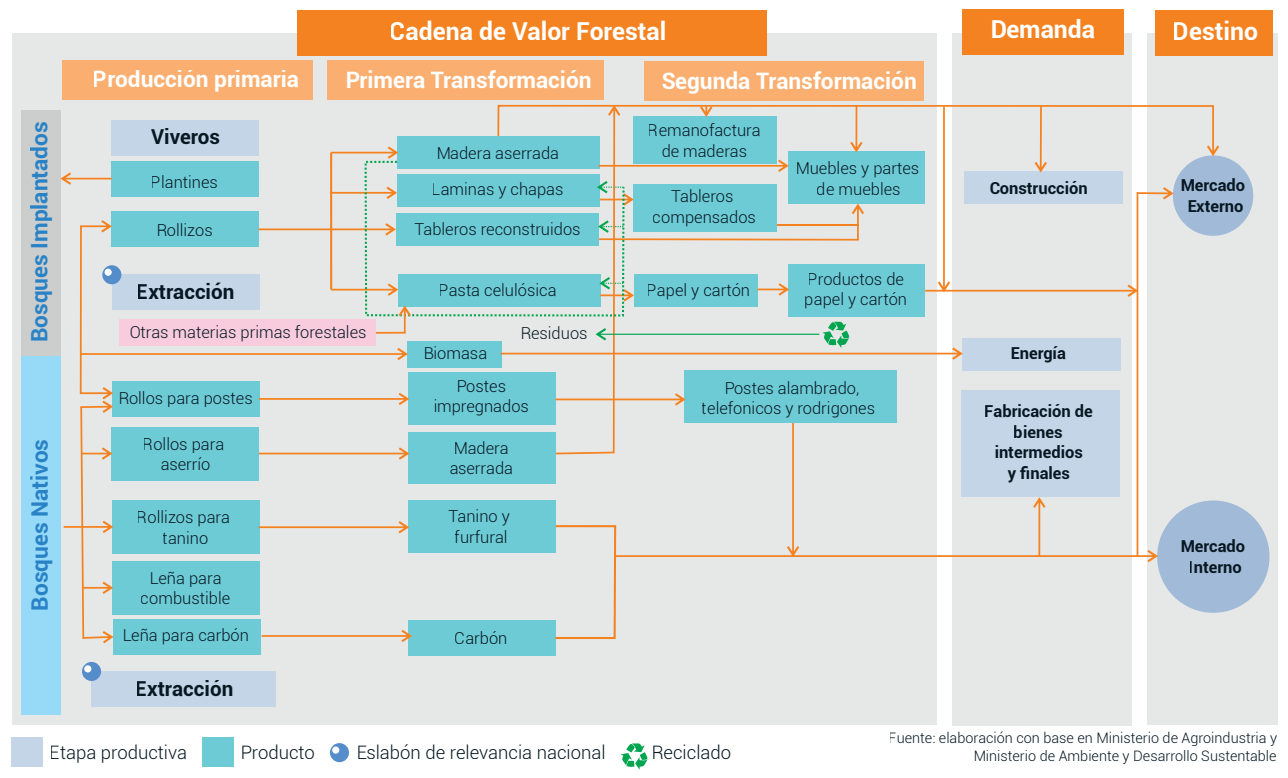

Figura 2. La cadena forestal en Argentina

Fuente: Subsecretaría de Programación Económica [12]

En la provincia de Santiago del Estero, la cadena foresto-industrial es más reducida que la exhibida en la figura 2. La producción primaria se basa en la producción de rollizos y leña. En cuanto a la primera transformación, predomina la producción de madera aserrada, postes, durmientes y carbón; la segunda comprende la elaboración de muebles, aberturas, implementos para la ganadería y otros productos de carpintería (figura 3). Todos los productos se destinan a abastecer el mercado interno a excepción del carbón, que se exporta a la Unión Europea, el NAFTA y el Mercosur.

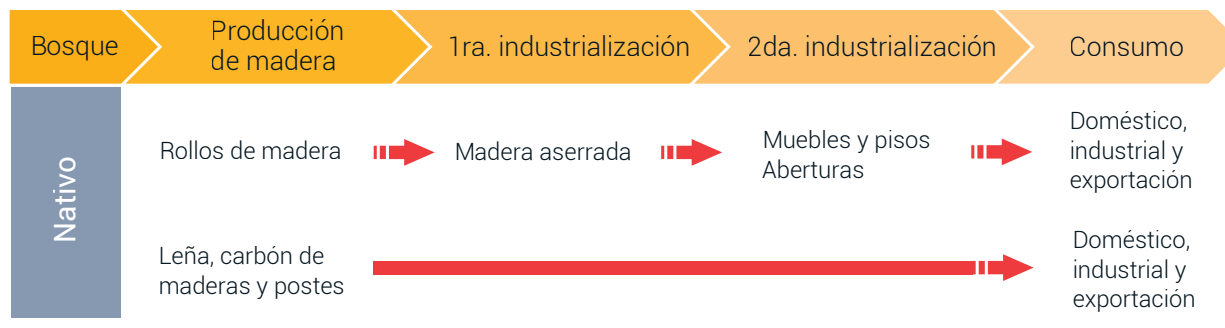

Figura 3. La cadena foresto-industrial de Santiago del Estero

Fuente: Ministerio de Producción-UISDE [6]

Utilizando información primaria y secundaria, se procedió a describir cada uno de los eslabones presentes en la cadena foresto-industrial santiagueña. 


\subsubsection{Fuente de la materia prima}

El primer eslabón en la cadena se da en el bosque nativo cuando los insumos provienen de este tipo de formación boscosa; mientras que, si el origen de la madera corresponde a las plantaciones, el primer eslabón se origina antes, con la obtención de semillas.

Los bosques implantados poseen una cadena con mayor cantidad de eslabones que la de los nativos, ya que esta se origina con la producción de la semilla mediante un mercado y con precios determinados. Los oferentes de semillas en Santiago del Estero son, principalmente, los bancos de germoplasmas (o bancos de semillas) y los productores primarios dedicados a la recolección de semillas de diversas especies. Estas semillas son demandadas por los viveros públicos y privados de la provincia, quienes las utilizan para la producción de plantines.

Los viveros estatales de la provincia de Santiago del Estero con fines productivos son los siguientes: Estación Experimental San Carlos (Departamento Banda), Estación Experimental Fernández (Departamento Robles) y Vivero Forestal General San Martín (Departamento Capital). Los dos primeros generan en conjunto una producción aproximada de 60.000 plantines de eucaliptos y 80.000 barbados de álamos. El vivero General San Martín, por su parte, genera una producción de 200.000 plantines de eucaliptos, 150.000 de paraíso, 10.000 barbados de salicáceas y 500.000 de otras especies forestales. En cuanto a los viveros de gestión privada, solo hay cuatro de carácter forestal certificados e inscritos en el Instituto Nacional de Semillas (Inase); se desconoce su producción.

Los bosques implantados son muy relevantes en el ámbito nacional, ya que cubren la mayor parte de la demanda de materia prima de las industrias de base forestal. Sin embargo, en el ámbito provincial no se observa la misma importancia.

Entre los años 2000 y 2016, Santiago del Estero forestó unas 1.832 hectáreas bajo el régimen de la Ley n. 25.080 de inversiones para bosques cultivados [2]; no se encontraron registros sobre plantaciones que no obedezcan a los beneficios de dicha ley. El algarrobo blanco (Prosopis alba) es la principal especie cultivada en la provincia (alrededor del $90 \%$ ), seguida en orden de importancia por el álamo (Populus sp).

La superficie total forestada en Santiago del Estero representa apenas el 0,15\% del área forestada en el país, lo que da indicios de la poca importancia que tienen los bosques implantados en el ámbito provincial. Actualmente, es inexistente la integración en la cadena foresto-industrial del eslabón primario con la producción de maderas y muebles para este tipo de bosques.

Los bosques nativos de la provincia, por su parte, suman cerca de 7 millones de hectáreas en diversos estados de conservación [1]. El aprovechamiento de 
estos se realiza principalmente de especies autóctonas como el quebracho colorado (Schinopsis quebracho colorado), el quebracho blanco (Aspidosperma quebracho blanco) y el algarrobo blanco (Prosopis alba).

El consumo de materia prima rolliza en los aserraderos y carpinterías de la provincia es muy diferente según las zonas: la zona norte consume el $77 \%$ del total; la zona centro, el $10 \%$; y la zona sur, el $13 \%$ restante [4].

La presión para obtener madera de los bosques nativos es muy grande, encontrándose Santiago del Estero entre las provincias con mayores tasas de deforestación del país, junto con Salta, Chaco y Formosa. Esta situación no es amortiguada por la presencia de bosques implantados, como ocurre en las provincias de la región mesopotámica.

\subsubsection{Producción primaria}

En la cadena foresto-industrial existe estrecha conexión entre el eslabón de la producción primaria y los siguientes, dado que los eslabones de la primera y segunda transformaciones utilizan la madera de origen local como fuente principal de aprovisionamiento [4].

La actividad extractiva es el eslabón predominante en la cadena en Santiago del Estero. La producción primaria proviene exclusivamente de los boques nativos; no se han registrado actividades de extracción provenientes de bosques implantados de la provincia. En este eslabón se realiza la extracción de madera, que es utilizada como tal o aplicada a posteriores procesos productivos. Los principales productos obtenidos de los bosques nativos son los rollizos (para postes y aserrío) y la leña (para combustible y carbón). La producción de postes y rodrigones se lleva a cabo dentro del monte con escaso grado de elaboración. Los rollos para aserrío se transportan a los establecimientos madereros, donde se realizan los procesos de primera y segunda transformación mecánica. En la provincia no se produce tanino, pero se estima que esta industria utiliza unas 40.716 toneladas de rollos de quebracho colorado cuyo origen es santiagueño [6].

La actividad forestal primaria se origina en los productores forestales u obrajeros, quienes son los encargados de realizar las extracciones del bosque, o bien de adquirir la materia prima a sus propietarios. Estos últimos constituyen el eslabón más débil de la cadena, ya que tienen elevada dependencia de los obrajeros, muy poca capacidad de negociación y nulo poder de mercado; poseen emprendimientos familiares de pequeña dimensión, participan fuera del circuito formal y presentan características de subsistencia. Los obrajeros detentan el manejo real del bosque y establecen los 
vínculos con los demás actores de la cadena [12]. A excepción de los departamentos de Copo y Alberdi, hay poca evidencia de que se verifique una integración vertical entre este eslabón productivo y los siguientes, ya que quienes desarrollan la actividad extractiva venden su producción a los aserraderos [12].

En algunas localidades del noreste provincial, como Monte Quemado y Campo Gallo, existen grandes productores forestales que poseen, además, aserraderos o carpinterías, con lo que se muestra cierto grado de integración vertical en la cadena forestal. No se ha observado lo mismo en los departamentos de Banda, Capital y Loreto.

\subsubsection{Primera transformación de la madera}

La etapa de la primera transformación de la madera en Santiago del Estero se basa en la elaboración de carbón, postes y madera aserrada. La provincia es la segunda productora de carbón vegetal de Argentina después de Chaco, con una producción anual que representa el $27 \%$ del total nacional [1]. En cuanto a los postes y rodrigones, la provincia aporta un poco más del 50 \% de la producción de postes provenientes de los bosques nativos del país. Las principales especies utilizadas son el quebracho colorado y el itín (Prosopis kuntzei), para los cuales no se registran procesos posteriores de agregado de valor [12].

La actividad industrial de la madera aserrada se reduce al proceso mecánico del corte del rollo con sierra y al secado de la madera (que, en la mayoría de los casos, se hace de forma natural). Los productos de primera transformación generados por los aserraderos son, en orden de importancia, durmientes para ferrocarril, varillas para alambrado, tablas, tirantes, alfajías, tablones, tirantillos y vigas; todos estos de bajo valor agregado.

La mayor producción de este eslabón de la cadena se concentra en la cercanía de los recursos forestales, particularmente en los departamentos Copo y Alberdi, al norte de la provincia. La producción de madera de primera transformación generada por los aserraderos fue de $27.330 \mathrm{~m}^{3}$ en 2015 (tabla 1).

Tabla 1. Producción de madera de primera transformación en Santiago del Estero. Año $2015\left(\right.$ en $\left.\mathrm{m}^{3}\right)$

\begin{tabular}{cccccc}
\hline Producto & Durmientes & Varillas & Tablas & Tirantes & Otros \\
\hline Volumen & 16.446 & 5.780 & 3.249 & 764 & 1.091 \\
\hline
\end{tabular}

Fuente: Ministerio de Agroindustria [4] 
De la madera nativa utilizada, el $92 \%$ corresponde a quebracho blanco y colorado, ambos destinados, en su mayoría, a la producción de durmientes; y un 7 \% es algarrobo, cuyo destino principal es la fabricación de muebles.

\subsubsection{Segunda transformación de la madera}

La industria de segunda transformación de la madera, y particularmente el sector de muebles, se encuentra atomizada: más del 90 \% de las empresas son de pequeño porte. En Santiago del Estero, este eslabón de la cadena consiste en la obtención de productos tales como muebles y partes de ellos, implementos ganaderos, y pisos. Los implementos de ganadería hacen referencia a mangas, bretes, cargaderos, casillas de operarios, tranqueras, etc. La tabla 2 resume los volúmenes producidos en 2015 según el tipo de producto. Como puede observarse, la fabricación de muebles, que incluye aberturas, es del $86 \%$; un $13 \%$ es implementos de ganadería; y el resto de la producción corresponde a pisos de madera. De estos volúmenes de producción, la zona norte de la provincia aporta la mitad de los implementos de ganadería y el $75 \%$ de los pisos, mientras que las tres zonas participan en la producción de muebles en proporciones similares. Los productos de carpintería tienen mayor valor agregado y, por lo tanto, mayor valor comercial, pero la producción es menos importante en términos físicos.

Tabla 2. Volumen de producción de remanufacturas en Santiago del Estero, año $2015\left(\right.$ en $\left.\mathrm{m}^{3}\right)$

\begin{tabular}{cc}
\hline Producto & Cantidad \\
\hline Muebles & 2.534 \\
\hline Implementos de ganadería & 384 \\
\hline Pisos & 16 \\
\hline
\end{tabular}

Fuente: Ministerio de Agroindustria [4]

\subsubsection{Subproductos}

Los datos de las entrevistas revelaron la ausencia de gestión de los residuos. Los desechos madereros representan alrededor del $30 \%$ del recurso forestal utilizado. Los subproductos del aserradero son aserrín, viruta, despuntes y costaneros de las maderas de quebracho y algarrobo; estos tienen un valor potencial de mercado para su uso en diferentes procesos industriales. Los aserraderos no cuantifican los subproductos generados en el proceso, salvo por los despuntes y costaneros de quebracho 
que se venden como leña para los fabricantes de carbón, los cuales son de fácil colocación en el mercado. El aserrín y la viruta se intercambian a ladrilleros por ladrillos, se regalan o se queman para mantener limpio el aserradero.

\subsubsection{Demanda}

La producción de la foresto-industria argentina puede destinarse a los mercados interno o externo. En el segundo, Argentina es un país proveedor de bienes como pasta celulósica y tableros de madera, destinados a la industrialización en países de mayor desarrollo relativo; y es importador de productos más elaborados, principalmente papel y muebles. Una de las barreras para acceder a los mercados externos es el problema de escala, que ha generado en los últimos años una pérdida de las incipientes exportaciones. Según el Informe de cadenas de valor del sector [12], en 2014 el consumo aparente de madera aserrada en Argentina fue de 3.474.000 toneladas, con un elevado crecimiento (del 75,7 \%) en los últimos 5 años, inducido en primer lugar por la demanda interna generada por la industria de la construcción.

A diferencia de lo que sucede con la madera destinada a la construcción, los muebles se venden directamente al público, por lo que el aumento de los ingresos personales es una de las principales causas de mayor demanda de estos productos; con lo cual se evidencia una elasticidad-ingreso positiva para dichos productos.

En Santiago del Estero, la demanda de productos elaborados tiene sus particularidades según el producto que se trate. Para los durmientes, la estructura de mercado se corresponde con un monopsonio u oligopsonio en el que existen uno o pocos demandantes cuyo destino final es el sistema ferroviario nacional; y no hay estrategias activas de comercialización de estos productos, como sucede también con las tablas producidas en la provincia. En cuanto a los muebles, en general la producción está destinada al mercado local salvo en Loreto, donde una parte de la producción se destina al mercado nacional [5].

El potencial del sector maderero reside en su vinculación con un importante nicho de mercado: el sector de la construcción. El Informe productivo provincial [19] destaca que la industria de la construcción registró un fuerte crecimiento absoluto (años 2010-2015) según exhiben los permisos de edificación, con un crecimiento del $141 \%$. Esto implica un incremento de la demanda de carpintería de obra y de servicios de instalación de mobiliarios y aberturas. 


\subsection{Ambiente organizacional}

A nivel provincial, se identificaron numerosas instituciones oficiales y organizaciones no gubernamentales involucradas con el sector de la industria forestal. La Facultad de Ciencias Forestales de la Universidad Nacional de Santiago del Estero (FCF-UNSE) forma técnicos en aserrado y carpintería industrial, con lo que se aporta mano de obra calificada, además de preparar profesionales de la ingeniería. Esta institución, además, contribuye con investigación científica junto con la Estación Experimental de la Universidad Católica de Santiago del Estero (EE-Fernández). También existen entidades gremiales y empresariales como el Colegio de Graduados en Ciencias Forestales, la Asociación de Productores de la Industria Forestal (APIF), la Federación Obrera Santiagueña de la Industria Forestal (Fosif), la Unión Industrial de Santiago del Estero (UISDE), la Cámara de Comercio e Industrias de Santiago del Estero (CCISGo) y la Cámara de la Industria Maderera (Cimse). El marco institucional se completa con el Ministerio de Producción, Recursos Naturales, Forestación y Tierras en tanto organismo estatal, y con una organización intermedia, la Mesa Foresto-Industrial (Mefise).

Entre las instituciones nacionales con filiales provinciales se pueden mencionar la Subsecretaría de Desarrollo Foresto-Industrial (SSDFI); el Instituto Nacional de Tecnología Agropecuaria; la Subsecretaría de Agricultura Familiar Delegación Santiago del Estero; y el Instituto Nacional de Tecnología Industrial (INTI) Centro Regional Santiago del Estero. Este último cuenta con la dependencia Madera y Muebles, que realiza trabajos técnicos y de asesoría al sector.

El rol de la Mefise es fundamental, dado que es un ámbito de trabajo interdisciplinario e interinstitucional. Esta fue creada en 2013, con el propósito de promocionar y brindar asesoría respecto de medidas conducentes a potenciar el desarrollo foresto-industrial de Santiago del Estero, tales como el desarrollo de políticas de financiamiento fiscal, laboral, de innovación y desarrollo que beneficien al sector; el fortalecimiento de la competitividad del sector en un entorno innovador; y la promoción de diversas formas de asociativismo.

\subsection{Marco normativo}

Santiago del Estero cuenta con una serie de herramientas legales de promoción, tales como leyes nacionales y provinciales que promueven el desarrollo forestoindustrial. Las leyes nacionales n. 26.331 de Presupuestos Mínimos de Protección Ambiental de los Bosques Nativos y 25.080 de Promoción de las Inversiones en Bosques Cultivados, otorgan financiamiento a los productores forestales. La Ley 
Nacional n. 27.349 de Apoyo al Capital Emprendedor, por su parte, apoya y fomenta el desarrollo local de diferentes actividades productivas. La Ley Provincial n. 6942 de Ordenamiento Territorial de Bosques Nativos, la n. 6841 de Conservación y Uso Múltiple de las Áreas Forestales de Santiago del Estero, y principalmente la n. 6750 de Promoción y Desarrollo Industrial, dan cuenta de la decisión del Gobierno de dar un fuerte impulso al sector foresto-industrial. Sin embargo, estas herramientas de política aún no han tenido el impacto esperado en el sector maderero.

\subsection{Caracterización de los actores directos de la cadena de la madera y el mueble}

Con los datos del CNA [4] y los resultados de las entrevistas in situ se delineó una breve caracterización de los establecimientos que conforman el eslabón produccióntransformación-comercialización de la cadena de la madera y el mueble en Santiago del Estero.

\subsubsection{Perfil de los establecimientos}

Los resultados del CNA revelaron la existencia de 206 establecimientos en la provincia, entre aserraderos e industrias de manufactura [4]. Del análisis de las entrevistas se infiere que el 31 \% son aserraderos, situados principalmente en La Banda, Monte Quemado y Campo Gallo. Las carpinterías representan el 53 \% y elaboran muebles, aberturas y otros productos (pérgolas, bancos, techos, escaleras, tarimas y palets). De ese $53 \%$, un tercio funciona como carpintería de forma exclusiva; el resto tiene el aserradero integrado a la misma y se ubica en Capital, Loreto y Monte Quemado (figura 4).

Un $17 \%$ de la muestra representa otro tipo de establecimientos dedicados a diversas actividades madereras proveedoras de bienes y servicios: elaboración de puertas placa para viviendas, trabajos de tornería y artesanías, reparación de muebles, fábrica de virutas y corralones (compra y venta de madera a medida). La gran mayoría son industrias que exceden ampliamente los diez años de antigüedad; sin embargo, un tercio del total es de reciente instalación y no supera los cinco años de trayectoria. 


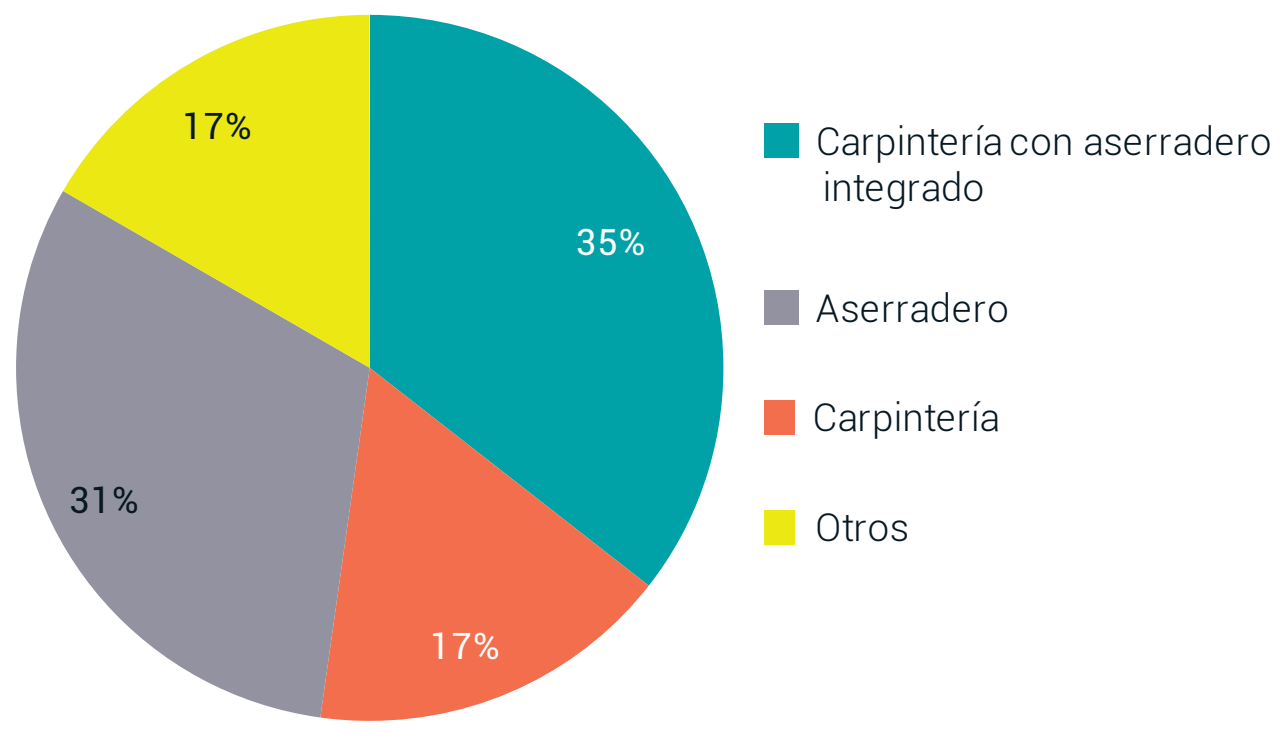

Figura 4. Establecimientos madereros de Santiago del Estero Fuente: elaboración propia

En cuanto al tamaño de los establecimientos, definido por el volumen anual de producción, el 96 \% produce una cantidad inferior a los $940 \mathrm{~m}^{3}$ (microempresas), mientras que el $4 \%$ restante (pequeñas empresas) produce entre 940 y $4720 \mathrm{~m}^{3}$. La zona norte aporta el mayor porcentaje de producción, como se observa en la figura 5. El rendimiento promedio (producción/materia prima) es del orden del 35 \% [4].

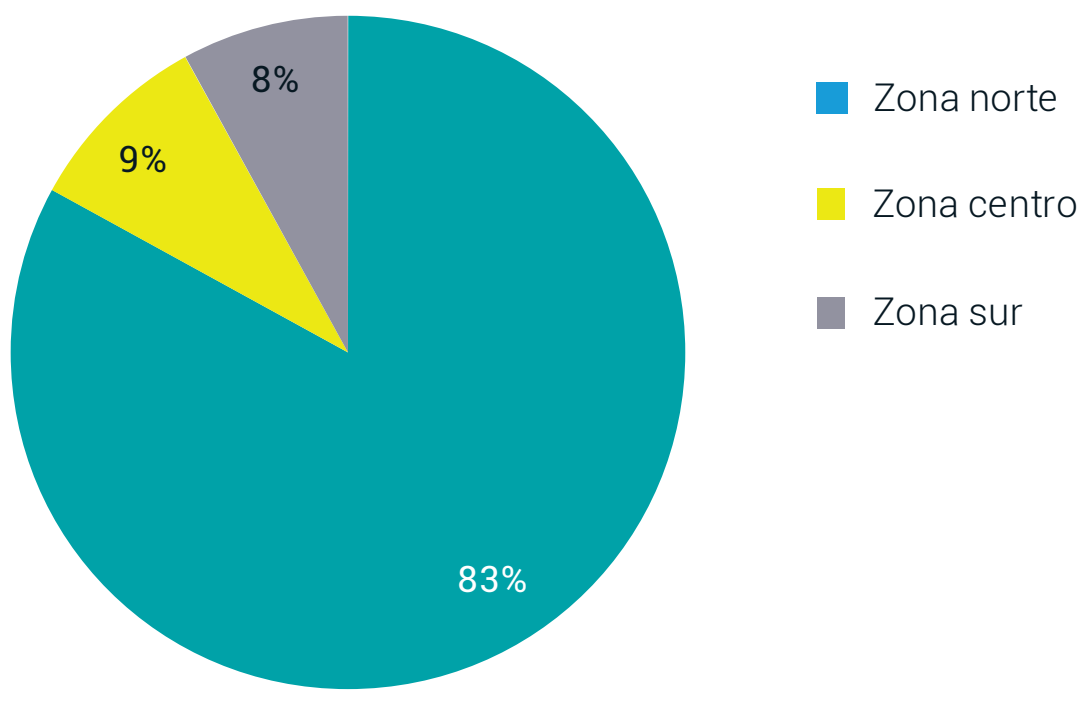

Figura 5. Porcentaje de la producción maderera por zonas Fuente: Ministerio de Agroindustria [4] 


\subsubsection{Materia prima utilizada}

En general, los rollizos de madera nativa son de calidad regular y diámetros variados, debido a la falta de manejo del recurso. Existen dificultades para el abastecimiento de los rollos de algarrobo en particular, a causa de una marcada disminución de su stock. En las industrias forestales de la zona norte predomina el consumo de maderas de quebracho blanco y colorado; y a medida que se avanza hacia la zona sur de la provincia, si bien el consumo de materia prima es marcadamente inferior, las proporciones del uso de algarrobo aumentan.

Los establecimientos de primera transformación (aserraderos de La Banda, Monte Quemado y Campo Gallo) emplean como materia prima solamente la madera de quebracho colorado, quebracho blanco y algarrobo, proveniente del bosque nativo (figura 6).

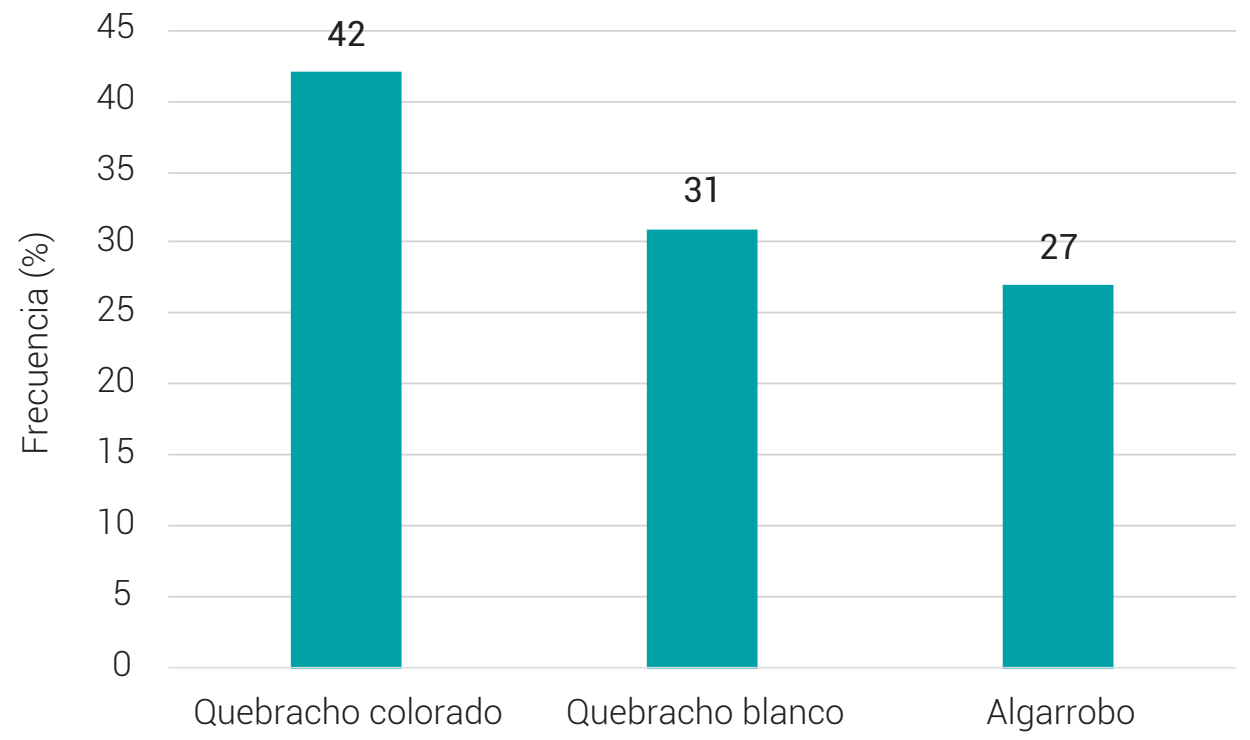

Figura 6. Especies forestales utilizadas por los aserraderos Fuente: elaboración propia

Por su parte, las industrias de segunda transformación (carpinterías de Loreto, Monte Quemado y Capital) utilizan principalmente el algarrobo como materia prima. Para la fabricación de muebles, las carpinterías ubicadas en los centros de consumo de Capital y La Banda emplean maderas de origen extraprovincial, como el cedro (Cedrela sp) y el pino (Pinus elliottii), además del algarrobo de origen local (figura 7). Asimismo, recurren a insumos sustitutos de la madera maciza, tales como los 
tableros de fibra de media densidad (MDF). Loreto representa un núcleo de carpinterías especializado en la fabricación de muebles de algarrobo, exclusivamente.

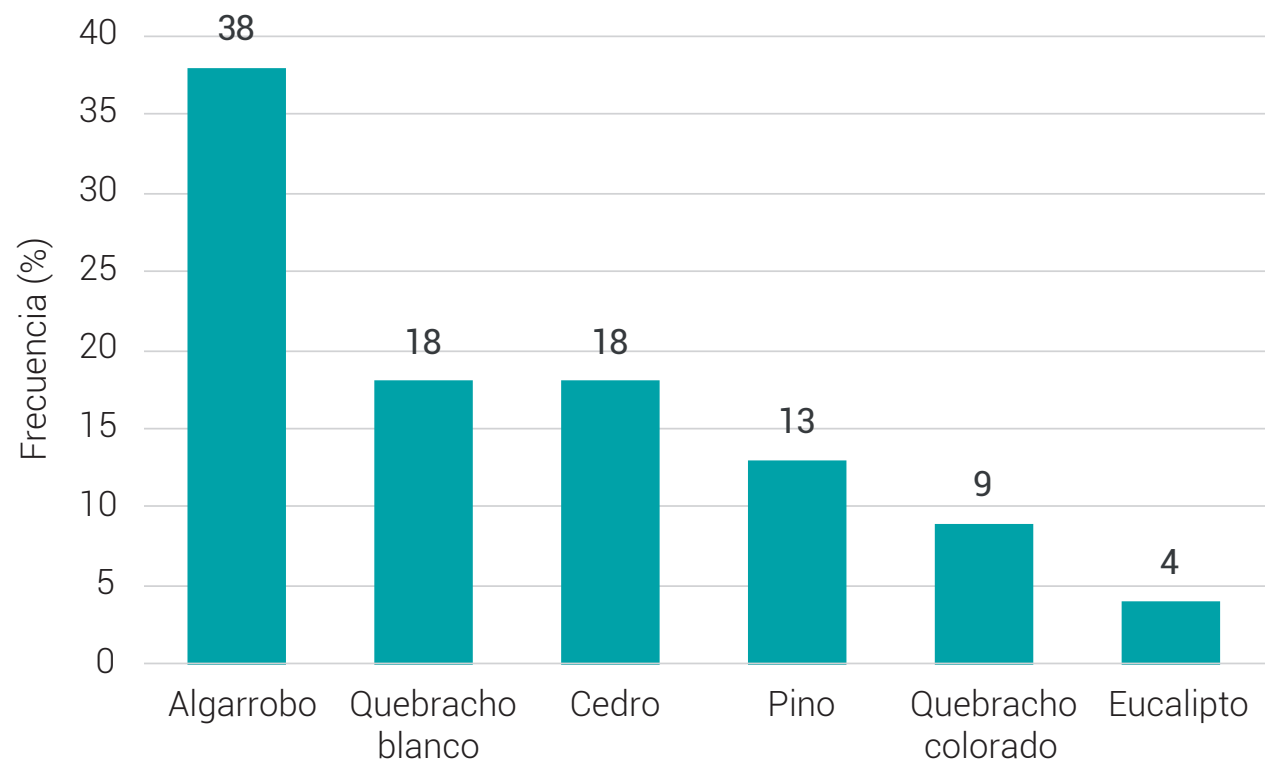

Figura 7. Especies forestales utilizadas por las carpinterías

Fuente: elaboración propia

\subsubsection{Equipamiento}

La potencia instalada del parque de maquinaria suma unos $7171 \mathrm{Hp}$ : de estos, el 43 \% corresponde a la zona norte de la provincia, que potencialmente tiene la mayor capacidad de producción; le siguen las zonas centro y sur en menor medida. El consumo eléctrico estimado por año es de 3.915.617 kWh [4].

Según el CNA [4], la capacidad ociosa promedio del equipamiento ronda el $34 \%$ y la zona centro ostenta el valor más alto. Los datos de las encuestas confirmaron estos resultados: la tasa de utilización de la maquinaria es de grado bajo en La Banda; moderado en Capital; y alto en Loreto, Monte Quemado y Campo Gallo.

Los aserraderos utilizan la sierra sin fin con carro como principal. Los distintos tipos de máquinas empleadas tienen una antigüedad promedio de 50 años. A pesar de la obsolescencia, en líneas generales, el equipamiento se encuentra en buen estado de mantenimiento. 


\subsubsection{Empleo}

Las industrias madereras de la provincia generan empleo directo de 970 puestos de trabajo. La zona norte es la que posee la mayor cantidad de empleados, con un promedio de 6 personas por establecimiento; las industrias de la zona centro, 5 personas; y las de la zona sur, 3 personas. La zona norte presenta el mayor valor de productividad por empleado [4].

Según las encuestas, el nivel de capacitación del personal es alto para el 56 \% de los establecimientos visitados (principalmente en Monte Quemado y Loreto), y moderado para un $34 \%$ del total. El resto posee trabajadores sin adiestramiento, para los que debe advertirse que se trata de industrias de mucha antigüedad, en las que era esperable encontrar mano de obra capacitada.

\subsubsection{Comercialización}

El análisis de las entrevistas reveló que la comercialización tiene como destino casi exclusivo el mercado local. No se observaron operaciones de venta de maderas o muebles al exterior; los industriales desconocen las normativas internacionales.

El $57 \%$ de las industrias de primera y segunda transformación de la madera comercializa toda su producción a clientes particulares; el $17 \%$ es proveedor de empresas exclusivamente, mientras que el $26 \%$ atiende un mix de clientes que incluye particulares, empresas y, de modo excepcional, el Estado provincial (figura 8).

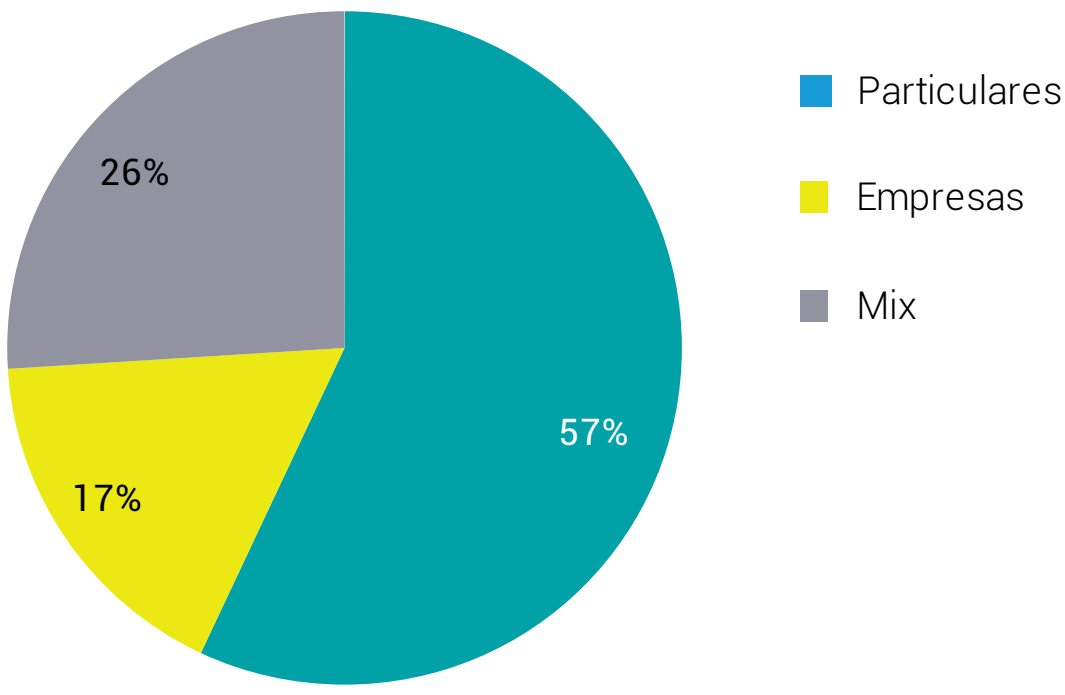

Figura 8. Destino de las ventas

Fuente: elaboración propia 
La comercialización en el eslabón de la primera industrialización no posee estrategias directas de ventas ya que los industriales no realizan publicidad ni promoción alguna de sus productos, sino que se manejan con su cartera de clientes tradicionales (que generalmente son intermediarios) y no atraen a nuevos consumidores. Las estrategias de venta en el eslabón de la segunda industrialización son diferentes según la localización de las carpinterías: para el caso de Capital y La Banda, las más grandes poseen algún espacio de exposición de sus productos o locales específicos para la comercialización de muebles y aberturas; pero las más pequeñas (que son la mayoría) no tienen producción para exponer. Esta carencia de espacio destinado a la venta está presente en el $100 \%$ de las carpinterías encuestadas en Loreto y Monte Quemado.

Las encuestas mostraron que el comportamiento de las ventas en los tres últimos años (2014-2016) resultó muy variable entre los establecimientos. Un 40 \% considera que sus ventas permanecieron en el mismo nivel. Para un 36 \%, hubo una reducción significativa (de manera predominante en Loreto, La Banda y Monte Quemado); y para el resto (principalmente en Capital), las ventas ascendieron en el periodo considerado.

La contracción de las ventas que se señala a nivel local coincide con el desempeño a nivel nacional, que describe una caída de las ventas de madera y muebles en torno al 20 \% frente a 2015, según la oıмyм [3]. Este comportamiento podría tener explicaciones en aspectos macroeconómicos (como la alta inflación y el desempleo imperante en el país), así como en problemas de estructura productiva al interior de las empresas. Hay factores endógenos que facilitan o dificultan el sostenimiento del ritmo de ventas. En este sentido, el buen nivel de capacidad operativa, la variedad de maderas utilizadas, la diversificación de productos y la diversidad de clientes y nichos de mercado son características de las industrias de Capital, y explicarían el desempeño ascendente de sus ventas.

El pronóstico de ventas para los próximos tres años (2017-2019) es alentador para el $37 \%$ de los casos, que sostiene expectativas positivas. Sin embargo, el $39 \%$ tiene incertidumbre sobre el futuro y el $24 \%$ restante concibe un escenario poco optimista, dada la desfavorable situación actual de las variables macroeconómicas del país.

\subsubsection{Competencia en el sector}

Durante las entrevistas se evaluaron aspectos de competitividad relacionados con factores internos de la empresa, tales como el reconocimiento de competidores, la percepción del grado de competencia y las estrategias para competir. La gran 
mayoría (83 \%) reconoció la influencia y el accionar de la competencia en el sector; sin embargo, no todos los empresarios la perciben en igual grado. El 36 \% calificó su actividad como altamente competitiva (especialmente en Loreto); el $43 \%$, como moderada o normal; y el resto la consideró baja. Las estrategias aplicadas para enfrentar la competencia son, en orden decreciente, calidad del producto, experiencia en el rubro, precios bajos, seriedad en la entrega, diseño y diferenciación, y buena atención al cliente.

El mayor grado de competencia se verifica en el caso de la cadena del mueble: por un lado, los muebles de madera maciza compiten en el mercado local con muebles más livianos elaborados con tableros MDF, que están ganando participación por ser fáciles de transportar y tener un precio menor; y por otro, las aberturas de madera maciza están perdiendo participación en el mercado, ya que compiten con los bienes sustitutos de aluminio o chapa.

Se pudo observar una situación particular en Loreto: existe alta competencia entre las carpinterías locales, generada por una "guerra de precios", lo que ocasiona que el mueble se venda finalmente a un precio que apenas supera los costos fijos de producción. Otra competencia importante para estas carpinterías son los muebles que ingresan de la provincia de Chaco, que se diferencian por ser de mejor calidad (en diseño y acabado) y precios más bajos que los muebles santiagueños, ya que aquellos tienen un fuerte apoyo estatal.

\subsubsection{Relaciones interinstitucionales}

Resulta indudable la importancia de la articulación del sector privado con agentes gubernamentales e instituciones vinculadas a la ciencia y al desarrollo tecnológico. Los procesos de articulación influyen de forma positiva en los resultados innovadores de las industrias, que no dependen solamente de la relación entre insumos y productos, sino también de las interacciones entre los actores involucrados.

De las entrevistas se infiere que existe una cultura individualista en los industriales de la madera, dada su reducida participación e integración con instituciones intermedias vinculadas con el sector forestal. Asimismo, su vinculación con los centros educativos de investigación y formación profesional es limitada.

Por otra parte, a pesar de que existe un marco institucional y normativo adecuado para promover el desarrollo del sector, son escasos los industriales interesados en acogerse a las ventajas que otorgan las leyes de promoción vigentes. El alto grado de informalidad en que estos desarrollan sus actividades productivas y comerciales les impide cumplir con los requisitos que la ley impone para acceder a los beneficios. 


\subsection{Análisis FODA de la cadena de la madera y el mueble}

Con base en la información obtenida en las encuestas, entrevistas y bibliografía disponible, se resumieron los factores críticos del funcionamiento de la cadena de la madera y el mueble. La aplicación del análisis FODA permitió identificar restricciones y cuellos de botella, así como desafíos y oportunidades (tabla 3).

Tabla 3. Análisis FODA de la cadena de la madera y el mueble de Santiago del Estero

\begin{tabular}{|c|c|}
\hline Fortalezas & Oportunidades \\
\hline $\begin{array}{l}\text { F1. Amplia trayectoria en el mercado local con sufi- } \\
\text { ciente experiencia en la actividad }\end{array}$ & $\begin{array}{l}\text { O1. Potencial de aprovechamiento de residuos de la } \\
\text { industria }\end{array}$ \\
\hline $\begin{array}{l}\text { F2. Importante cantidad de empresas de la industria } \\
\text { maderera }\end{array}$ & $\begin{array}{l}\text { O2. Incremento de la demanda de productos madere- } \\
\text { ros por aumentos significativos en el sector local } \\
\text { de la construcción }\end{array}$ \\
\hline F3. Capacidad instalada disponible & O3. Posibilidades de mayor diversificación \\
\hline F4. Aserraderos ubicados próximos a la materia prima & $\begin{array}{l}\text { O4. Posibilidades de producción asociativa entre } \\
\text { aserraderos y carpinterías }\end{array}$ \\
\hline $\begin{array}{l}\text { F5. Posibilidades de formación y capacitación técnica } \\
\text { y profesional }\end{array}$ & $\begin{array}{l}\text { O5. Entorno institucional concientizado con la promo- } \\
\text { ción industrial }\end{array}$ \\
\hline $\begin{array}{l}\text { F6. Aceptable optimismo en las perspectivas futuras } \\
\text { del sector }\end{array}$ & $\begin{array}{l}\text { O6. Potencial de vinculación intersectorial alentada } \\
\text { por la creación de instituciones y ONG del sector }\end{array}$ \\
\hline $\begin{array}{l}\text { F7. Moderada integración tecnológica (aserrade- } \\
\text { ro-carpintería) }\end{array}$ & $\begin{array}{l}\text { 07. Alta disponibilidad de biomasa forestal proveedo- } \\
\text { ra de madera }\end{array}$ \\
\hline $\begin{array}{l}\text { F8. Variedad de oferta dirigida a diferentes segmentos } \\
\text { y nichos de mercado }\end{array}$ & $\begin{array}{l}\text { O8. Existencia de leyes sobre el manejo del bosque } \\
\text { nativo e implantado }\end{array}$ \\
\hline \multirow[t]{3}{*}{$\begin{array}{l}\text { F9. Elaboración de productos de madera de modera- } \\
\text { da calidad }\end{array}$} & $\begin{array}{l}\text { 09. Tecnologías disponibles para el manejo sustenta- } \\
\text { ble del bosque nativo }\end{array}$ \\
\hline & $\begin{array}{l}\text { O10.Adecuado entorno científico-tecnológico para el } \\
\text { apoyo a la industria }\end{array}$ \\
\hline & O11.Existencia de leyes de promoción industrial \\
\hline Debilidades & Amenazas \\
\hline $\begin{array}{l}\text { D1. Insuficiente capacitación de recursos humanos, } \\
\text { tanto en producción como en oficios específicos y } \\
\text { en gestión }\end{array}$ & $\begin{array}{l}\text { A1. Información oficial y privada insuficiente, dispersa } \\
\text { y divergente }\end{array}$ \\
\hline $\begin{array}{l}\text { D2. Escasa inversión en infraestructura y maquinaria } \\
\text { industrial }\end{array}$ & $\begin{array}{l}\text { A2. Alta competencia de provincias vecinas en el } \\
\text { mercado local de muebles }\end{array}$ \\
\hline $\begin{array}{l}\text { D3. Falta de ideas innovadoras para ofrecer productos } \\
\text { con mayor agregado de valor }\end{array}$ & $\begin{array}{l}\text { A3. Informalidad en la producción primaria de la } \\
\text { madera }\end{array}$ \\
\hline D4. Restricciones en la producción a escala & $\begin{array}{l}\text { A4. Competencia entre los muebles y aberturas de } \\
\text { madera por productos sustitutos }\end{array}$ \\
\hline $\begin{array}{l}\text { D5. Alto grado de informalidad del mercado que } \\
\text { propicia la competencia desleal. }\end{array}$ & $\begin{array}{l}\text { A5. Ausencia de normativas que impulsen el agregado } \\
\text { de valor en origen. }\end{array}$ \\
\hline D6. Dificultades en la provisión de materia prima local & $\begin{array}{l}\text { A6. Insuficientes políticas públicas para el financia- } \\
\text { miento de inversiones }\end{array}$ \\
\hline
\end{tabular}




\begin{tabular}{|c|c|}
\hline Debilidades & Amenazas \\
\hline $\begin{array}{l}\text { D7. Baja calidad de la madera proveniente del bosque } \\
\text { nativo }\end{array}$ & $\begin{array}{l}\text { A7 Falta de programas que financien la capacitación y } \\
\text { asistencia técnica }\end{array}$ \\
\hline D8. Excesivo individualismo en las actuaciones & A8. Adversa situación macroeconómica \\
\hline D9. Limitado acceso al crédito & A9. Escasa oferta adaptada a la demanda \\
\hline $\begin{array}{l}\text { D10. Escasa participación del sector en las licitaciones } \\
\text { de obras públicas }\end{array}$ & $\begin{array}{l}\text { A10. Bajo nivel de coordinación en la promoción indus- } \\
\text { trial }\end{array}$ \\
\hline $\begin{array}{l}\text { D11. Baja articulación entre empresas e instituciones } \\
\text { de I\&D }\end{array}$ & $\begin{array}{l}\text { A11. Falta de políticas estatales para incluir al sector en } \\
\text { las licitaciones públicas }\end{array}$ \\
\hline D12. Desconocimiento del mercado externo & \\
\hline
\end{tabular}

Fuente: elaboración propia

\section{Conclusiones y recomendaciones}

La cadena foresto-industrial con origen en los bosques nativos tiene continuidad en todos los eslabones productivos; mientras que aquella con origen en las plantaciones es discontinua y se manifiesta solo en el eslabón inicial con una muy baja participación, retomando la cadena en la segunda transformación para la elaboración de muebles y partes de ellos, en la cual el origen de la madera utilizada es extraprovincial.

La cadena de la madera y el mueble de Santiago del Estero posee todas las características propias de una cadena productiva, que marcan la diferencia con las cadenas de valor: los actores son independientes, la producción es traccionada por la oferta, la competencia está basada principalmente en los precios, los productos elaborados son básicos y con escasa diferenciación, la relación entre los actores es baja y acontece de manera informal, y los flujos de información son escasos.

El alto grado de informalidad con el que se desarrollan las actividades productivas, así como la fuerte cultura individualista en las conductas de los actores directos, no permite generar procesos de integración. Se identifican debilidades y amenazas en todo el eslabonamiento de la cadena, desde la producción hasta la comercialización; no obstante, existen fortalezas y oportunidades para potenciar y capitalizar, tales como la capacidad industrial disponible y las posibilidades de capacitación profesional, entre las primeras, y la vigencia de adecuadas leyes de manejo forestal y el entorno de ciencia y tecnología propicio, entre las segundas.

Identificados los factores críticos, se debería avanzar en la definición de objetivos estratégicos ofensivos y defensivos de la cadena, y en el diseño de planes concertados de acción, tanto desde el interior de las empresas como desde el Estado y otras 
instituciones. La cooperación entre los diferentes actores facilitaría la integración, con lo que mejorarían el desempeño futuro y la competitividad de la cadena.

\section{Referencias}

[1] Ministerio de Ambiente y Desarrollo Sustentable, Superficie de bosque nativo según el Ordenamiento Territorial de Bosques Nativos, 2018 [En línea]. Disponible en: http://mapas. ambiente.gob.ar/archivos/web/MapAMB/file/metadata/ley_bosques/OTBN_12012018.pdf

[2] Ministerio de Agroindustria, Inventario nacional de plantaciones forestales, 2017 [En línea]. Disponible en: http://datos.agroindustria.gob.ar/dataset/inventario-nacional-deplantaciones-forestales-por-superficie

[3] Observatorio de la industria de la madera y el mueble (огмум), Actualidad de la cadena de valor foresto-industrial. Informe omym, 2016 [En línea]. Disponible en: https://es.scribd.com/ document/347850346/Informe-OIMyM-2016

[4] Ministerio de Agroindustria, Censo nacional de aserraderos: Informe del relevamiento censal en la provincia de Santiago del Estero. Buenos Aires: Ministerio de Agroindustria, 2017, 27p [En línea]. Disponible en: https://www.agroindustria.gob.ar/sitio/areas/ss_desarrollo_foresto_ industrial/censos_inventario/_archivos/censo//000000_Provincia\%20de\%20Santiago\%20 del\%20Estero\%20(Abril\%202017).pdf

[5] R. Sánchez-Ugalde, Informe final de consultoría para la instalación de un centro de capacitación. Buenos Aires: Programa de Sustentabilidad y Competitividad Forestal, BID 2853 OC/AR, 2012, 92p.

[6] Ministerio de Producción-UISDE, Plan Estratégico Industrial Santiago del Estero 2020. Santiago del Estero: Ministerio de Producción, Unión Industrial de Santiago del Estero, 2013, 196p [En línea]. Disponible en: http://uisde.com.ar/documentos/PEI-SE_VERSION-IMPRENTA.docx

[7] V. Cuevas-Reyes, "Análisis del enfoque de cadenas productivas en México”. Textual, n. 56, pp. 83-94, Jul. - Dic. 2010 [En línea]. Disponible en: http://www.chapingo.mx/revistas/textual/ contenido.php?id_articulo=1260\&id_revistas $=2$

[8] S. E. Cayeros-Altamirano, F. J. Robles-Zepeda y E. Soto-Ceja, "Cadenas productivas y cadenas de valor". Educateconciencia, vol.10, n¹1, pp.6-12, abr-jun. 2016 [En línea]. Disponible en: http://tecnocientifica.com.mx/educateconciencia/index.php/revistaeducate/article/ view/129 
[9] A. M. G. de Castro, "Análise diagnóstica de cadeias produtivas". En: V. A. Hoeflich. Cadeia produtiva do negócio florestal. Curitiba: UFPR; Colombo: Embrapa Florestas, 2007, 18p [En línea]. Disponible en: http://fcf.unse.edu.ar/archivos/posgrado/Aula\%2005_An\%C3\%A1lise\%20 Diagn\%C3\%B3stica.vh2008Tania.rev..pdf

[10] J. Federico, Análisis de diagnóstico tecnológico sectorial. Madera y muebles. Buenos Aires: Secretaria de Planeamiento y Políticas, Ministerio de Ciencia, Tecnología e Innovación Productiva, 2013, 62p [En línea]. Disponible en: http://www.mincyt.gob.ar/adjuntos/archivos/000/043/0000043765.pdf

[11] G. Idígoras, Análisis tecnológicos y prospectivos sectoriales. Prospectiva tecnológica al 2025 del complejo foresto-industrial. Buenos Aires: Secretaria de Planeamiento y Políticas, Ministerio de Ciencia, Tecnología e Innovación Productiva, 2016, 76p [En línea]. Disponible en: http://www.mincyt.gob.ar/adjuntos/archivos/000/047/0000047562.pdf

[12] Subsecretaría de Programación Económica (SSPE), Informes de Cadenas de Valor. Forestal, papel y muebles, Año 1, n.ำ14. Buenos Aires: Subsecretaría de Programación Económica, Ministerio de Hacienda y Finanzas Públicas, 2016, 60p [En línea]. Disponible en: www.economia.gob.ar/peconomica/docs/SSPE_Cadenas\%20de\%20valor_Forestal,\%20papel\%20 y\%20muebles.pdf

[13] J. Rodríguez-Zúñiga, M. González-Guillén y E. Valtierra-Pacheco, "Análisis estratégico de la cadena productiva forestal en la región de la Reserva de la Biosfera de la Mariposa Monarca”. Madera y Bosques, vol. 24, n. ${ }^{\circ}$ 1, ene. 2018. doi: https://doi.org/10.21829/myb.2018.2411404

[14] A. C. Padilla-Puerta, Propuesta de formulación de la cadena productiva forestal del Departamento de Arauca. Arauca: Universidad Nacional de Colombia, 2014, 206p [En línea]. Disponible en: http://bdigital.unal.edu.co/42017/1/7709576.2014.pdf

[15] K. Salas-Navarro, "Análisis de la Cadena de Abastecimiento del Sector Madera y Muebles de la ciudad de Barranquilla". Scientia et Technica, vol. XVI, n. ${ }^{\circ}$ 49, pp. 229-238, dic. 2011 [En línea]. Disponible en: http://www.redalyc.org/articulo.oa?id=84922625039

[16] J. L. Lima, Estudio de la caracterización de la cadena de producción y comercialización de la industria forestales: estructuras, agentes y prácticas. Santiago: ODEPA, Ministerio de Agricultura, Gobierno de Chile, 2016, 106p [En línea]. Disponible en: https://www.odepa.gob.cl/wp-content/uploads/2017/12/InformelndustriaForestal2016.pdf

[17] F. R. David, Conceptos de administración estratégica, 12. ${ }^{a}$ edición. México: Pearson Educación, 2008, pp. 221-225. 
[18] H.Ponce-Talancón, "La matriz FODA: alternativa de diagnóstico y determinación de estrategias de intervención en diversas organizaciones”. Enseñanza e Investigación en Psicología, vol. 12, n. ${ }^{\circ}$ 1, pp. 113-130, ene-jun. 2007 [En línea]. http://www.redalyc.org/articulo.oa?id=29212108

[19] Subsecretaría de Programación Económica (SSPE), Informes Productivos Provinciales. Santiago del Estero, Año 1, n. 9. Buenos Aires: Subsecretaría de Programación Económica, Ministerio de Hacienda y Finanzas Públicas, 2016, 36p [En línea]. Disponible en: https://www. economia.gob.ar/peconomica/dnper/fichas_provinciales/Informe_Productivo_Santiago_ del_Estero.pdf 